sedimenting particles contained a complete succinic oxidase system but could not oxidize glucose. One can conclude that all particles, irrespective of size, contain the succinic oxidase system, while the oxidation of glucose requires soluble glycolytic enzymes which are present only in the supernatant fractions.

On treating $S_{1}$ with ammonium sulphate, it was found that virtually all the protein was precipitated between 50 and 65 per cent saturation. The precipitate, obtained by mixing 100 c.c. $S_{3}$ with 200 c.c. saturated ammonium sulphate, was centrifuged down and tested as follows. A small quantity was neutralized with sodium carbonate and reduced with dithionite. Whereas the bands of $b_{1}$ and $a_{1}$ were very strong, $a_{2}$ could not be detected. The bulk of the precipitate was dialysed against distilled water to remove ammonium sulphate, when a clear brownish liquid was obtained. It was examined spectroscopically at room temperature and also, after mixing with glycerol, at liquid air temperature ${ }^{b}$. Cytochromes $b_{1}$ and $a_{1}$ were very distinct and greatly intensified at the lower temperature, but $a_{2}$ could not be seen in either case. The dialysed preparation oxidized succinate but did not take up any oxygen with glucose.

Although there is considerable evidence that cytochrome $a_{2}$, which occurs in various bacteria, functions as cytochrome oxidase ${ }^{1,7}$, the present experiments suggest that the oxidation of succinate by extracts of $A$. aerogenes can proceed without it. On the other hand, if $a_{2}$ is highly active, it is very possible that a concentration sufficient for a measurable oxidation may be invisible spectroscopically.

Molteno Institute, University of Cambridge.

1 Tissières, A., Biochem. J., 50, 279 (1951).

${ }^{2}$ Wiggert, W. P., Silverman, M., Utter, M. F., and Werkman, C. H., 14, $179(1940)$

${ }^{3}$ Haas, E., J. Biol. Chem., 148, 481 (1943). "Eichel, B., Wainio, W. W., and Person, P., J. Biol. Chem., 183, 89
(1950).

${ }^{5}$ Smith, L., and Stotz, E., Fed. Proc., 9, 230 (1950).

${ }^{6}$ Keilin, D., and Hartree, E. F., Nature, 164, 254 (1949).

'Negelein, E., and Gärischer, W., Biochem. Z., 268, 1 (1934).

\section{Cyclical Transmission of Plasmodium berghei in the Laboratory}

CyClical transmission of $P$. berghei has been successfully carried out under experimental conditions. A batch of laboratory-bred A. quadrimaculatus and $A$. stephensi fed on February 15 on a hamster infected eight days previously with the Kasapa strain of $P$. berghei. The hamster showed numerous gametocytes in the peripheral blood at the time of the blood meal. Dissections of mosquitoes' stomachs showed a 50 per cent infection-rate. On February 25, twenty $A$. stephensi and twenty-two A. quadrimaculatus of this batch bit and fully engorged themselves on a month-old white rat. The salivary glands of these mosquitoes were dissected out in Ringer-glucose and injected into the same animal on February 26.

Daily blood smears were taken. On the morning of March 2, that is, six days after biting, three young parasites were found in a blood smear. They were small rings showing distinct vacuoles. Smears and sections made from different organs have not as yet revealed exo-erythrocytic parasites; but two mature schizonts of hæmatogenous origin were found in the bone marrow. It may therefore be deduced that the invasion of the blood cells occurred approximately on the fourth day after sporozoite inoculation. Work on the primary development of $P$. berghe $i$ is continuing.

We wish to thank Prof. J. Rodhain and Dr. I. H. Vincke for providing the strain of $P$. berghei; we also acknowledge the help and encouragement given by Prof. H. E. Shortt, Prof. G. Macdonald and Dr. P. C. C. Garnham. One of us (M. Y.) is indebted to the Milner Research Fund in Tropical Medicine for a grant.

\section{YokxI}

Hebrew University, Jerusalem.

$$
\text { W. J. WALL }
$$

Ross Institute,

London School of Hygiene and Tropical Medicine. March 14.

${ }^{1}$ Yoeli, M., and Wall, W. J., Nature, 168, 1078 (1951).

"Vincke, I. H., and Lips, M., Ann. Soc. Belge Med. Trop., 39, 1605 (1950).

\section{Self-cure in Infections of Trichostrongylus retortæformis and its Causation}

THE phenomenon of self-cure in nematode infections has been reported from time to time: Using infections of Trichostrongylus retortoeformis in rabbits, we have begun a study of some of the circumstances in which this phenomenon occurs.

The course of an infection established by the administration of a single dose of larvæ varies. Either it is terminated abruptly after a few weeks, or no such sudden self-cure takes place and the fæcal eggcount falls very gradually over a period of many months, the rate of fall itself decreasing meanwhile. The causes for this difference between individual host animals is not yet clear; but the age of the host and genetic differences appear to be involved.

When a new infection is superimposed on an existing one in an animal that is not capable of the selfcure mechanism the effect is additive, and if the dose of larvæ is sufficiently large death results.

In rabbits capable of self-cure, the duration of an infection bears an inverse relation to its size and, if the number of worms is very small, self-cure never occurs at all. When we superimpose a new infection on a light one of this nature, that has been patent for some time, the effect depends on the size of the superimposed dose of larvæ. When this is small the effect is additive : the fæcal egg-count rises, but there is no self-cure. When a moderate-sized dose is given, the egg-count rises after a fortnight-the normal prepatent period-and self-cure thereupon follows immediately, the egg-count reaching zero in three weeks from the time of re-infection. When a massive dose is administered, self-cure is complete within a week.

From these results we conclude that a certain quantity of worm material must be present to evoke self-cure. Thus, in the light initial infection, not enough is present; when the small dose is added there is still not enough, and even the moderate dose does not provide enough until the worms have grown to maturity. Only with the massive re-infecting dose do we achieve the necessary quantity at once.

Veterinary Laboratory, J. F. MICHEL

Weybridge, Surrey.

Dec. 31 